\title{
Presentación del trabajo de investigación y defensa del Diploma de Estudios Avanzados
}

\author{
Francisco Tomas Aguirrea, Francisca Gil Latorre ${ }^{a}$,

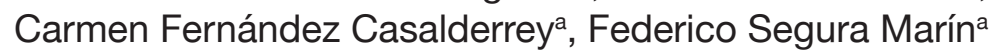

\begin{abstract}
a Unidad Docente de Medicina de Familia y Comunitaria de Valencia.
\end{abstract}

Correspondencia:

Francisca Gil Latorre.

C/ Juan de Garay n ${ }^{\circ} 21$,

46017-Valencia.

Telf.: 686323261,

e-mail: gil_fra@gva.es.

Recibido el 9 de enero de 2008.

Aceptado para su publicación el 23 de enero de 2008.

\section{RESUMEN}

Las normativas para las "lecturas", "defensas" o exposiciones para la obtención del Diploma de Estudios Avanzados (DEA) están claramente definidas en cada Universidad y en los reglamentos de régimen interno de cada Departamento Universitario que desarrolla programas de doctorado, pero solamente hacen referencia a los aspectos formales, tales como plazos, duraciones, composición de tribunales, etc. y no se recogen de forma explícita los contenidos que deberán ser expuestos en las mismas. La demanda, en nuestra Unidad Docente de MFyC, tanto de residentes como de médicos de familia en dicho asesoramiento, nos ha llevado a la necesidad de elaborar un documento que recoja los consejos prácticos y las orientaciones para la presentación del trabajo del Diploma de Estudios Avanzados.

Palabras clave. Educación de Postgrado en Medicina.

\section{ABSTRACT}

Presentation of research studies to obtain the Advanced Studies Diploma.

In Spain scientific production in primary care has increased at a remarkable rate in the last few years. Spain now occupies fourth place amongst the countries with the highest rate of recent production. Castilla-La Mancha also occupies fourth place amongst the Spanish autonomous communities in scientific production in Primary Care, as regards production per million inhabitants. Temporal progression of the impact factors and citations is positive and has shown an increase in recent years. The mean impact factor of the journals in which primary care articles are published is increasing every year. This means that Primary Care professionals in Castilla-La Mancha are publishing in higher quality journals more frequently.

Key Words. Education Medical Graduate.

\section{INTRODUCCIÓN}

Los requisitos de presentación del Diploma de Estudios Avanzados ${ }^{1}$ (DEA) son:

1. Un análisis del desarrollo del programa realizado, y los conocimientos que se han adquirido.

2. Exposición del trabajo de investigación (TI) realizado y de los resultados más relevantes.

3. Explicación de la relación existente entre los trabajos de investigación y la tesis que se pretende desarrollar.

La inexistencia de normas escritas que sirvan de ayuda a la preparación de la presentación del DEA, motivan las consideraciones incluidas en este artículo. El objetivo planteado será revisar los puntos más importantes para organizar la exposición de los TI y la defensa del DEA. Para ello, se tendrá en cuenta, por una parte, cómo explicar los contenidos y la presentación formal ${ }^{2,3}$ del Tl y cómo exponer de forma clara los aspectos diferenciadores entre dicha presentación y la defensa del diploma, y, por otra parte, que el doctorando disponga de una sencilla guía metodológica para prepararla de forma eficiente.

\section{CÓMO EXPLICAR LOS CONTENIDOS DEL TRABAJO DE INVESTIGACIÓN}

1. Introducción: enunciado del problema, hipótesis y objetivos. 
2. Métodos: con el tipo de estudio, justificando el diseño, población de estudio, muestra y detalle de las variables.

3. Resultados: que sirvan de base para las conclusiones.

4. Discusión: evidencias que fundamentan las conclusiones.

5. Conclusiones: numerarlas y hacer énfasis en cada una.

\section{PRESENTACIÓN FORMAL DEL TRABAJO DE INVESTIGACIÓN}

Se debe ensayar con el tutor y algún compañero con el fin de controlar el tiempo y extraer críticas constructivas. Para comenzar, el doctorando se debe presentar (nombre, apellidos, departamento etc.). No se debe leer lo escrito en la diapositiva: el lenguaje hablado no es igual que el escrito, es más coloquial, utiliza frases cortas. Las pausas reemplazan a la puntuación. Utilizar la modulación de la voz y gestos para enfatizar. Se recomienda no leer los títulos de cada apartado (Introducción, Métodos, etc.), por ejemplo, tras formular el objetivo, podemos decir algo como "para ello utilizamos un estudio transversal", en vez de decir "Métodos". Los medios audiovisuales adecuados sirven para apoyar o ampliar la palabra, nunca para reemplazarla.

\section{LA DEFENSA PÚBLICA DEL DEA}

Las normas para las "lecturas", "defensas" o exposiciones para la obtención del DEA están definidas en cada Universidad y en los reglamentos de cada Departamento que desarrolle programas de doctorado, pero solamente hacen referencia a los aspectos formales, tales como plazos, duraciones, composición de tribunales, etc. No se recogen de forma explícita los contenidos que deberán ser expuestos en las mismas.

\section{LA PREPARACIÓN DEL DEA}

En primer lugar es necesario recopilar las materias y conocimientos adquiridos. Para ello, se puede emplear el plan de estudios oficial del departamento. En la actualidad, la mayoría de Universidades disponen de sus propios espacios web con información al respecto. De no ser así, será necesario dirigirse a la comisión de investigación para obtener dicha información. En este plan se detalla la composición de los cursos, la troncalidad u optatividad de los mismos, sus objetivos o contenidos, así como información sobre el profesorado. A continuación, es recomendable el empleo de los materiales utilizados en la defensa del TI: esquemas, resúmenes, tablas, guiones, etc., seleccionando los más adecuados para la defensa del DEA.

\section{ORGANIZACIÓN DE LA PRESENTACIÓN DEL DEA}

La organización propuesta se articula como sigue:

- Presentación del Programa de Doctorado cursado.

- Presentación de las asignaturas cursadas.

- Exposición de la aplicación de los contenidos de los cursos para el TI.

- Presentación (resumida) del TI.

- Exposición de las líneas futuras de trabajo abiertas.

Además, partiendo de los 30 minutos de exposición,

\begin{tabular}{|c|c|}
\hline Cursos & Aspectos Destacados \\
\hline Diseños Epidemiológicos en Investigación & $\begin{array}{l}\text { - Condiciones diferenciales de la investigación } \\
\text { - Necesidad de utilizar variables operativas } \\
\text { - Principales diseños utilizados }\end{array}$ \\
\hline Derecho Médico Aplicado & $\begin{array}{l}\text { - Normas jurídicas que regulan el ejercicio de la Medicina } \\
\text { - Conocimientos necesarios para resolver problemas }\end{array}$ \\
\hline $\begin{array}{l}\text { Epidemiología y Prevención de Enfermedades } \\
\text { Tramsmisibles }\end{array}$ & $\begin{array}{l}\text { - Principales grupos de enfermedades transmisibles } \\
\text { - Vacunas, su eficacia efectividad y nuevas tecnologías }\end{array}$ \\
\hline Epidemiología Genética y Molecular & $\begin{array}{l}\text { - Integración de las medidas de la variabilidad genética con las de los } \\
\text { demás determinantes no genéticos en los estudios epidemiológicos } \\
\text { - Desarrollar estrategias de prevención }\end{array}$ \\
\hline Investigación Criminal & $\begin{array}{l}\text { - Escena del crimen } \\
\text { - Trabajo de laboratorio }\end{array}$ \\
\hline Medicina Legal del Trabajo & $\begin{array}{l}\text { - Aspectos médico-legales de la Medicina del Trabajo } \\
\text { - Conocimientos necesarios para el médico de atención primaria de salud }\end{array}$ \\
\hline Problemas de Psiquiatría Forense & $\begin{array}{l}\text { - Conceptos básicos de la Psiquiatría Legal y Forense. } \\
\text { - Repercusiones de la enfermedad mental en los distintos campos } \\
\text { del Derecho }\end{array}$ \\
\hline Trabajo de Investigación & $\begin{array}{l}\text { - DEMANDA DE LA ANTICONCEPCION DE URGENCIA EN UN CENTRO } \\
\text { DE ATENCION CONTINUADA }\end{array}$ \\
\hline
\end{tabular}

Tabla 1. Cursos desarrollados (ejemplo) y breve reseña de los aspectos más representativos de los mismos 


\begin{tabular}{l|l}
\hline Cursos & Aplicabilidad al Trabajo de Investigación \\
\hline Diseños Epidemiológicos en Investigación & DISEÑO Y ANÁLISIS DE RESULTADOS \\
\hline Derecho Médico Aplicado & ASPECTOS ÉTICOS \\
\hline Epidemiología y Prevención de Enfermedades & CONOCIMIENTO EPIDEMIOLÓGICO EN EL MEDIO Y \\
Transmisibles & PLANTEAMIENTO DE INTERVENCIÓN COMUNITARIA \\
\hline Epidemiología Genética y Molecular & (específico - no relación directa) \\
\hline Investigación Criminal & (específico - no relación directa) \\
\hline Medicina Legal del Trabajo & CONOCIMIENTOS MÉDICO-LEGALES EN A. P. \\
\hline Problemas de Psiquiatría Forense & ANÁLISIS DE REPERCUSIONES LEGALES \\
\hline
\end{tabular}

Tabla 2. Ejemplo de aplicación de los contenidos para el Trabajo de Investigación.

tiempo asignado en la mayoría de normativas que regulan el DEA, se propone una distribución aproximada del mismo como sigue. Igualmente, con el fin de hacerlo más práctico, resumimos una muestra del esquema organizativo propuesto de la defensa pública real de un DEA en un departamento universitario.

Presentación del programa de doctorado (aproximadamente 2 minutos)

La presentación del programa en la defensa del DEA es obviada frecuentemente por el alumno. Se da por sentado que todos conocen y han cursado el mismo programa, pero no es siempre así. En muchos casos los departamentos ofertan más de un programa de doctorado, por lo que una buena manera de iniciar el aspecto formal de la exposición es realizar dicha presentación junto a la personal. Se presentará el programa enunciando su título oficial completo, el departamento que lo desarrolla y, si procede, la sección departamental que lo imparte, valorándose en cada caso si es conveniente mencionar al director y al profesorado del mismo.

En nuestro ejemplo: La doctoranda R. S. realiza la defensa pública de los conocimientos adquiridos y trabajos realizados para la obtención del Diploma de Estudios Avanzados en La Facultad de Medicina y Odontología de la Universitat de València, en el Departamento de Medicina Preventiva y Salud Pública, Sección Departamental de Medicina, Programa de Doctorado Salud Pública y Medicina Legal.

Presentación de las asignaturas cursadas (aproximadamente 5 minutos)

La mayoría de los cursos de doctorado presentan una oferta amplia que supera el número de créditos mínimos necesarios. El doctorando selecciona las asignaturas troncales y optativas que ha decidido cursar para obtener el DEA. Además de enumerarlas, podría ser conveniente exponer cuáles fueron las motiva- ciones para dicha elección. A continuación, de forma muy breve, se presentan las líneas básicas (objetivos o contenidos principales) de cada uno de los cursos desarrollados; también se pueden emplear las guías docentes de los departamentos.

La oferta docente del programa de nuestro ejemplo era de 18 cursos diferentes. La doctoranda expone los cursos que desarrolló y una breve reseña de los aspectos más representativos de los mismos (tabla 1).

Aplicación de los contenidos para el TI (aproximadamente 5 minutos)

Tras esta breve exposición, el doctorando extrae los aspectos específicos aprendidos y que ha podido aplicar de manera concreta en la realización de su TI. No se trata de repetir el apartado anterior, sino de recoger, preferentemente en forma de oraciones cortas, las aplicaciones prácticas de los contenidos teóricos a las distintas fases de su trabajo; desde la planificación, pasando por el diseño, hasta el análisis de las conclusiones. En la tabla 2 se muestra un ejemplo de la aplicación de los contenidos.

Presentación resumida del TI (aproximadamente 15 minutos)

La presentación pública del trabajo de investigación, en la mayoría de los casos, ya ha tenido lugar previamente. El doctorando ha defendido con anterioridad su investigación y ha sido juzgado y calificado por un tribunal del departamento. En la defensa del DEA no se debe caer en la equivocación de repetir exactamente dicha exposición. El doctorando debe conducirse presentando su TI dentro del marco del programa, es decir, como otro curso realizado. Se debe indicar al tribunal el día en que se defendió públicamente el trabajo, referenciar al director y aprovechar para realizar públicamente los agradecimientos al resto de personas que hayan podido colaborar en la investi- 
gación. Debe ponerse a disposición del tribunal para las aclaraciones que le sean solicitadas sobre el TI y, seguidamente, hacer una exposición más somera del trabajo, es decir, debe hacer énfasis en los objetivos, el diseño y las conclusiones.

Exposición de Líneas Futuras de trabajo (aproximadamente 3 minutos)

Como conclusión, es muy apropiado presentar las líneas de investigación nuevas que se derivan de las conclusiones del trabajo. Es recomendable enunciarlas de forma breve, a modo de títulos de trabajos futuros, y explicar el objetivo principal de cada una. Una manera ideal de finalizar este punto, y con él la parte formal de la defensa del DEA, es presentar como línea de trabajo el Proyecto de Tesis Doctoral que se vaya a llevar a cabo.

\section{BIBLIOGRAFÍA}

1. Universidad de Valencia. El diploma de estudios avanzados 2007. Disponible en: http://www.uv.es/ webuv/castellano/estudis/tercercicle.htm

2. Smith R. How not to give a presentation. BMJ 2000; 321:15701.

3. Albert Ros X, Gil Latorre F, Gutiérrez Sigler D. CICAP Investigación Clínica en Atención Primaria. Madrid: Luzán; 2004. p. 1-326. 